\title{
D-dimers at hospital admission for COVID-19 are associated with in hospital mortality independently of venous thromboembolism: Insight from a French multicenter cohort study
}

Richard Chocron ( $\nabla$ richard.chocron@gmail.com )

Assistance Publique - Hopitaux de Paris https://orcid.org/0000-0002-5498-8937

\section{Baptiste Duceau}

Paris University

Nicolas Gendron

Paris University

Nacim Ezzouhairi

Centre Hospitalier Universitaire de Bordeaux

Lina Khider

Hopital Europeen Georges Pompidou

Antonin Trimaille

Hopitaux universitaires de Strasbourg

Guillaume Goudot

Hopital Europeen Georges Pompidou

Orianne Weizman

Centre Hospitalier Universitaire de Nancy

Jean Marc Alsac

Hopital Europeen Georges Pompidou

Thibault Pommier

Centre Hospitalier Universitaire de Dijon

Olivier Bory

Hopital Europeen Georges Pompidou

Joffrey Cellier

Hopital Europeen Georges Pompidou

Aurélien Philippe

Hopital Europeen Georges Pompidou

\section{Laura Geneste}

Centre Hospitalier Universitaire Amiens-Picardie

lannis Ben Abdallah

Hopital Europeen Georges Pompidou 


\section{Vassili Panagides}

Assistance Publique Hopitaux de Marseille

\section{Salma El Batti}

Hopital Europeen Georges Pompidou

\section{Wassima Marsou}

Universite de Lille

Philippe Juvin

Hopital Europeen Georges Pompidou

\section{Antoine Deney}

Centre Hospitalier Universitaire de Toulouse

\section{Emmanuel Messas}

Hopital Europeen Georges Pompidou

\section{Sabir Attou}

Universite de Caen Normandie

\section{Benjamin Planquette}

Hopital Europeen Georges Pompidou

\section{Delphine Mika}

Assistance Publique - Hopitaux de Paris

\section{Pascale Gaussem}

Hopital Europeen Georges Pompidou

\section{Charles Fauvel}

Centre Hospitalier Universitaire de Rouen

\section{Jean-Luc Diehl}

Hopital Europeen Georges Pompidou

\section{Theo Pezel}

Assistance Publique - Hopitaux de Paris

\section{Tristan Mirault}

Hopital Europeen Georges Pompidou

\section{Willy Sutter}

Hopital Europeen Georges Pompidou

\section{Olivier Sanchez}

Hopital Europeen Georges Pompidou

\section{Guillaume Bonnet}

Hopital Europeen Georges Pompidou

\section{Ariel Cohen}

Assistance Publique - Hopitaux de Paris

David M. Smadja

Hopital Europeen Georges Pompidou 
Original research

Keywords: SARS-CoV-2, COVID-19, D-dimers, microvascular thrombosis, pulmonary embolism, deep venous thrombosis

Posted Date: August 28th, 2020

DOI: https://doi.org/10.21203/rs.3.rs-62363/v1

License: (c) (i) This work is licensed under a Creative Commons Attribution 4.0 International License.

Read Full License 


\section{Abstract}

Background: Coronavirus disease 2019 (COVID-19) has been associated with coagulation disorders, in particular high levels of D-dimers, and increased frequency of venous thromboembolism (VTE). We explore the association between D-dimers at admission and in-hospital mortality in hospitalized COVID19 patients with or without symptomatic VTE.

Methods: From February 26 to April 20, 2020, D-dimer level at admission and outcomes of patients hospitalized for COVID-19 in medical wards (in-hospital mortality or VTE) were retrospectively analyzed in a multicenter study in 24 French hospitals.

Results: Among 2878 patients enrolled in the study, 1154 (40.9\%) patients had D-dimer measurement at admission. A receiver operating characteristic (ROC) curve analysis identified D-dimer level above $1128 \mathrm{ng} / \mathrm{mL}$ as the optimum cutoff value to predict in-hospital mortality (Area Under the Curve of $64.9 \%$ $(95 \% \mathrm{Cl} 0.60-0.69)$ with a sensitivity of $71.1 \%(95 \% \mathrm{Cl} 0.62-0.78)$ and a specificity of $55.6 \%(95 \% \mathrm{Cl}$ $0.52-0.58$ ) that not differ in the subgroup of patients with VTE during hospitalization. Among 609 (52.8\%) patients with D-dimers level $<1128 \mathrm{ng} / \mathrm{mL}$ at admission, only $35(5.7 \%)$ deaths occurred during hospitalization. After adjustment, in a cox proportional hazard and logistic regression models, D-dimers above $1128 \mathrm{ng} / \mathrm{mL}$ at admission were also associated to a worth prognosis with a OR of $3.07(95 \% \mathrm{Cl}$ $2.05-4.69, \mathrm{p}<0.001)$ and an unadjusted hazard ratio of $2.11(95 \% \mathrm{Cl} 1.31-3.4, \mathrm{p}<0.01)$.

Conclusions: D-dimer level over $1128 \mathrm{ng} / \mathrm{mL}$ is a relevant predictive factor for in-hospital mortality in COVID-19 hospitalized patients in medical ward, regardless the occurrence of VTE during hospitalization.

\section{Introduction}

Infection with severe acute respiratory syndrome coronavirus 2 (SARS-CoV-2) is associated with various clinical respiratory syndromes, ranging from mild upper airway symptoms to progressive life-threatening viral pneumopathy $(1,2)$. Patients with severe coronavirus disease 2019 (COVID-19) have progressive hypoxemia inducing need of mechanical ventilatory support. One specific feature of COVID-19 is the induced-vascular disease. Ackermann et al. recently examined the morphologic and molecular features of lungs obtained during autopsy of patients who died from COVID-19 and evidenced abnormal angiogenic process inside lungs, in contrast to lungs from patients who died from influenza or age-matched and uninfected control lungs (3). COVID-19-induced vascular disease is also associated to an increased level of circulating endothelial cells (4). Moreover, plasma biomarkers of endothelial lesion are also predictive factors for future referral to intensive care unit (ICU), reinforcing the hypothesis of a COVID-19-associated vascular injury (5). SARS-CoV-2 virus has been shown to infect blood vessels and induce vascular damage (6) and fibrin deposits in lung but also in kidney has been found in vascular beds.

A high prevalence of venous thromboembolism (VTE), in particular pulmonary embolism (PE) has been observed in hospitalized COVID-19 patients (7-9). However, more than these macrothrombotic events, microvascular thrombosis in the lungs has been reported following autopsies, suggesting acute 
respiratory distress syndrome in COVID-19 (10-12). Thrombo-inflammatory process in pulmonary capillary vessels is probably the main actor of microthrombosis in lung capillaries that induces COVID-19associated coagulopathy (13), characterized by an increase in procoagulant factors such as fibrinogen, together with a strong increase of D-dimers at admission $(1,2,14)$. Level of D-dimers at admission has been associated to in-hospital mortality in several studies $(1,14,15)$, however the cut-off allowing deciphering patients with favorable and poor outcomes is still a matter of debate.

Using data from a large multicenter French case series, we aimed to identify a D-dimer cut-off at admission that could be a clear independent predictor of in-hospital mortality.

\section{Methods}

\section{Study Settings and Population}

From February 26 to April 20, 2020, all consecutive adult patients admitted to hospital with a diagnosis of SARS-CoV-2 infection were included in a retrospective multicentric (24 centers) observational study, which was initiated by the French Society of Cardiology (NCT04344327) and named the Critical COVID19 France (CCF) study (9). Following WHO criteria, SARS-CoV-2 infection was determined by positive results from real-time reverse transcriptase-polymerase chain reaction (RT-PCR) of nasal and pharyngeal swabs or lower respiratory tract aspirates (confirmed case) or was determined by typical imaging characteristics on chest computed tomography (CT) when laboratory testing was inconclusive (probable case) (16).

\section{Data Collection and outcome}

All data were collected by local investigators in an electronic case-report form via the REDCap software (Research Electronic Data Capture, Vanderbilt University, United States of America) hosted by a secured server from the French Institute of Health and Medical Research at the Paris Cardiovascular Research Centre. Patient baseline information included demographic characteristics, coexisting medical conditions, cardiovascular comorbidities and chronic medications. Clinical parameters and biological findings were recorded at admission. On the chest CT scan, the degree of pulmonary lesions with ground-glass opacities and areas of consolidation was categorized as low/moderate $(<50 \%$ involvement) or severe ( $>$ $50 \%$ involvement). The oral anticoagulation regimen at admission was categorized into two groups: 1) no anticoagulation 2) oral anticoagulant therapy with vitamin $\mathrm{K}$ antagonists or direct oral anticoagulants. The occurrence of symptomatic VTE during hospitalization included PE and/or deep vein thrombosis (DVT).

\section{Outcomes}

The primary outcome was in-hospital death to assess predictive performance of D-dimer level at admission in COVID-19 patients.

\section{Statistical Analysis}


Continuous data were expressed as mean ( \pm standard deviation (SD)) and categorical data as proportion. Continuous variables were compared using Mann-Whitney test and categorical variables were compared using Fisher exact test (17). We generated D-dimer level at admission receiver operating characteristic (ROC) curve for in-hospital mortality. We identified the optimal threshold of D-dimer level at admission using the Youden's $\mathrm{J}$ statistic. In the univariate analysis, patients were compared according to the optimal threshold of D-dimers at admission. In the multivariable analysis, we used logistic regression to assess the association between the level of D-dimers (as a categorical dependent variable dichotomized according to the optimal threshold) and platelet count, leukocyte count, or in-hospital mortality $(18,19)$. The model included as covariates: gender, age, cardiovascular comorbidities such as history of high blood pressure, history of malignancy (cancer in remission or active cancer), plasma creatinine level $(\mu \mathrm{moL} / \mathrm{L}), \mathrm{C}$-reactive protein $(\mathrm{mg} / \mathrm{L})$, the degree of pulmonary lesions with ground-glass opacities and areas of consolidation (dichotomized $<$ or $>50 \%$ ), the use of oral anticoagulant therapy and the occurrence of VTE during hospitalization. Cox proportional hazard $(\mathrm{PH})$ model with length of stay (days) as a time scale was used to investigate the relationships between the level of D-dimers (as a categorical dependent variable dichotomized according to the optimal threshold) and in-hospital mortality. The model was adjusted for the same potential confounders included in the logistic regression model. Kaplan-Meier method was used to represent Cox PH model results according to the level of D-dimers (as a categorical dependent variable dichotomized according to the optimal threshold). We used the log-rank test to compare the survival distributions according to the optimal threshold of D-dimers. We performed two sensitivity analysis: 1) to take into account the retrospective design and to avoid the bias due to censored data ( $n=268 / 1154(23.2 \%)$ ), we performed the same multivariable analysis in the population of patients who were discharged alive from hospital or dead in hospital (total patients analyzed $n=$ $886 / 1154(76.8 \%))$ and thus excluded patient with censored outcome. 2) We performed the D-dimer level at admission ROC curve only in the subgroup of patients with VTE during hospitalization $(n=127)$. We compared the area under the curve (AUC) of the two ROC curves using the Delong's test.

All analyses were 2 -sided and a $p$-value $<0.05$ was considered statistically significant. Statistical analysis was performed using R studio software (R Development Core Team (2019). R: A language and environment for statistical computing. R Foundation for Statistical Computing, Vienna, Austria).

\section{Results}

\section{Optimal D-dimer cut-off predicting in-hospital mortality did not differ in patients with or without VTE during hospitalization}

During the study period, a total of 2,878 consecutive patients who were hospitalized in medical ward for SARS-CoV-2 infection were included. At admission, 1154/2878 (40.1\%) patients had a mean (SD) age of 64.35 (16.63), and 59.8\% (690/1154) were female (Table 1). The optimum cut-off value for D-dimers at admission to predict in-hospital mortality was $1128 \mathrm{ng} / \mathrm{mL}$ according to ROC curve (Fig. 1) with a 
sensitivity of $71.1 \%(95 \% \mathrm{Cl} 0.62-0.78)$ and a specificity of $55.6 \%(95 \% \mathrm{Cl} 0.52-0.58)$, a positive predictive value of $15.8 \%$ ( $95 \% \mathrm{Cl} 0.13-0.19)$ and a negative predictive value of $94.3 \%$ ( $95 \% \mathrm{Cl} 0.92-$ 0.96). AUC for in-hospital mortality was $64.9 \%(95 \% \mathrm{Cl} 0.60-0.69)$. Listed in Table 1 are the initial clinical, biological and radiological characteristics and outcomes of the patients above and beyond the D-dimer cut-off of $1128 \mathrm{ng} / \mathrm{mL}$. At admission, 52.8\% (609/1154) patients had D-dimer levels below $1128 \mathrm{ng} / \mathrm{mL}$ and $47.2 \%$ (545/1154) over $1128 \mathrm{ng} / \mathrm{mL}$. Compared with patients with D-dimer levels below $1128 \mathrm{ng} / \mathrm{mL}$, patients with D-dimer levels $\geq 1128 \mathrm{ng} / \mathrm{mL}$ were older, had more high blood pressure and chronic kidney disease. Those patients had higher level of creatinine, C-reactive protein, fibrinogen, platelet and leukocyte counts, and a higher rate of severe parenchymal involvement on chest CT-scan. Moreover, those patients had a lower hemoglobin level and PT ratio. The in-hospital mortality rate (15.8\% vs $5.7 \%)$ and the mean duration of hospitalization (10.25 days (6.47) vs 8.75 days (5.83)) were significantly higher for COVID-19 patients with D-dimer level $\geq 1128 \mathrm{ng} / \mathrm{mL}$ at admission (Table 1 ). 
Table 1

Clinical, biological and radiological characteristics and outcomes according to optimal threshold of Ddimers at admission (<or $\geq 1128 \mathrm{ng} / \mathrm{mL}$ ).

\begin{tabular}{|c|c|c|c|c|}
\hline & $\begin{array}{l}\text { Overall } \\
\text { population }\end{array}$ & $\begin{array}{l}\text { D-dimers < } \\
1128 \mathrm{ng} / \mathrm{mL}\end{array}$ & $\begin{array}{l}\text { D-dimers } \geq \\
1128 \mathrm{ng} / \mathrm{mL}\end{array}$ & $\begin{array}{l}\text { p- } \\
\text { value }\end{array}$ \\
\hline & $n=1154$ & $n=609$ & $\mathrm{n}=545$ & \\
\hline Age, mean (SD) & 64.35(16.63) & 61.02(15.97) & 68.06(16.59) & $\begin{array}{l}< \\
0.001\end{array}$ \\
\hline \multicolumn{5}{|l|}{ Age range, $\mathrm{n}(\%)$} \\
\hline$(0,50]$ & $232(20.1)$ & $153(25.1)$ & $79(14.5)$ & $\begin{array}{l}< \\
0.001\end{array}$ \\
\hline$(50,60]$ & $210(18.2)$ & $137(22.5)$ & 73(13.4) & \\
\hline$(60,70]$ & $263(22.8)$ & $133(21.8)$ & $130(23.9)$ & \\
\hline$(70,80]$ & 224(19.4) & 106(17.4) & 118(21.7) & \\
\hline$(80,90]$ & 157(13.6) & $61(10.0)$ & $96(17.6)$ & \\
\hline$(90,110]$ & $65(5.6)$ & $17(2.8)$ & $48(8.8)$ & \\
\hline Male gender, n(\%) & $690(59.8)$ & $348(57.1)$ & $342(62.8)$ & 0.060 \\
\hline $\begin{array}{l}\text { Body Mass Index, mean } \\
\text { (SD) }\end{array}$ & $28.24(6.21)$ & $28.46(5.76)$ & $28.00(6.67)$ & 0.240 \\
\hline \multicolumn{5}{|l|}{ BMI range, $\mathrm{n}(\%)$} \\
\hline$(0,25]$ & $313(27.1)$ & $149(24.5)$ & 164(30.1) & 0.195 \\
\hline$(25,30]$ & $349(30.2)$ & 193(31.7) & $156(28.6)$ & \\
\hline$(30,66]$ & $320(27.7)$ & 174(28.6) & $146(26.8)$ & \\
\hline $\begin{array}{l}\text { Time from illness onset to } \\
\text { hospitalization in days, } \\
\text { mean (SD) }\end{array}$ & 7.12(4.76) & $7.14(4.61)$ & $7.10(4.92)$ & 0.902 \\
\hline \multicolumn{5}{|l|}{ Comorbidities } \\
\hline High blood pressure, n(\%) & $557(48.3)$ & $254(41.7)$ & $303(55.6)$ & $\begin{array}{l}<.001 \\
0.00\end{array}$ \\
\hline Diabetes, n(\%) & $259(22.4)$ & $126(20.7)$ & 133(24.4) & 0.316 \\
\hline Dyslipidemia, n(\%) & $314(27.2)$ & $152(25.0)$ & 162(29.7) & 0.075 \\
\hline History of stroke, n(\%) & $91(7.9)$ & $46(7.6)$ & $45(8.3)$ & 0.351 \\
\hline $\begin{array}{l}\text { Chronic kidney disease, } \\
\mathrm{n}(\%)\end{array}$ & $150(13.0)$ & $59(9.7)$ & $91(16.7)$ & <. 001 \\
\hline
\end{tabular}




\begin{tabular}{|c|c|c|c|c|}
\hline & $\begin{array}{l}\text { Overall } \\
\text { population }\end{array}$ & $\begin{array}{l}\text { D-dimers < } \\
1128 \mathrm{ng} / \mathrm{mL}\end{array}$ & $\begin{array}{l}\text { D-dimers } \geq \\
1128 \mathrm{ng} / \mathrm{mL}\end{array}$ & $\begin{array}{l}\mathrm{p}- \\
\text { value }\end{array}$ \\
\hline \multicolumn{5}{|l|}{ Malignancy, n(\%) } \\
\hline No cancer & $987(85.5)$ & $544(89.3)$ & $443(81.3)$ & $\begin{array}{l}< \\
0.001\end{array}$ \\
\hline Cancer in remission & $97(8.4)$ & $40(6.6)$ & $57(10.5)$ & \\
\hline Active cancer & $70(6.1)$ & $25(4.1)$ & $45(8.3)$ & \\
\hline Current smoker, n(\%) & 155(13.4) & $82(13.5)$ & 73(13.4) & 0.794 \\
\hline Atrial fibrillation, $\mathrm{n}(\%)$ & $129(11.2)$ & 71(11.7) & $58(10.6)$ & 0.855 \\
\hline \multicolumn{5}{|c|}{ Type of anticoagulation used at admission, $n(\%)$} \\
\hline no anticoagulation & 1025(88.8) & $539(88.5)$ & $486(89.2)$ & 0.978 \\
\hline DOACs & $74(6.4)$ & $40(6.6)$ & $34(6.2)$ & \\
\hline Vitamin $\mathrm{K}$ antagonist & $50(4.3)$ & $27(4.4)$ & $23(4.2)$ & \\
\hline Unfractionated Heparin & $5(0.4)$ & $3(0.5)$ & $2(0.4)$ & \\
\hline \multicolumn{5}{|l|}{$\begin{array}{l}\text { Use of oral anticoagulation } \\
\text { (DOACs or VKA), } n(\%)\end{array}$} \\
\hline yes & $124(10.7)$ & $67(11.0)$ & $57(10.5)$ & 0.906 \\
\hline no & 1025(88.8) & $539(88.5)$ & $486(89.2)$ & \\
\hline \multicolumn{5}{|l|}{ In hospital exploration } \\
\hline $\begin{array}{l}\text { Hemoglobin, } \mathrm{g} / \mathrm{dL} \text {, mean } \\
\text { (SD) }\end{array}$ & $13.21(1.96)$ & $13.57(1.75)$ & $12.80(2.10)$ & $\dot{0} 001$ \\
\hline $\begin{array}{l}\text { Platelets, } 10^{\wedge} 9 / L \text {, mean } \\
\text { (SD) }\end{array}$ & $222.46(100.28)$ & $208.34(80.98)$ & $238.31(116.31)$ & $\dot{0.001}$ \\
\hline $\begin{array}{l}\text { Creatinine level plasma, } \\
\text { micromol/L, mean (SD) }\end{array}$ & 98.61(99.85) & 87.87(79.77) & $110.60(117.22)$ & $\dot{0} 001$ \\
\hline $\begin{array}{l}\text { Aspartate } \\
\text { aminotransferase, UI/L, } \\
\text { mean (SD) }\end{array}$ & $56.18(83.32)$ & $51.53(64.91)$ & 61.37(99.74) & 0.050 \\
\hline $\begin{array}{l}\text { Leukocytes, } 10^{\wedge} 9 / \mathrm{L} \text {, mean } \\
\text { (SD) }\end{array}$ & 7.54(5.98) & $6.61(3.24)$ & $8.58(7.89)$ & $\dot{0.001}$ \\
\hline $\begin{array}{l}\text { Lymphocytes, } 10^{\wedge} 9 / \mathrm{L} \text {, mean } \\
\text { (SD) }\end{array}$ & $1.31(3.76)$ & $1.21(1.30)$ & $1.41(5.31)$ & 0.370 \\
\hline $\begin{array}{l}\text { C-reactive protein, } \mathrm{mg} / \mathrm{L} \text {, } \\
\text { mean (SD) }\end{array}$ & $91.52(76.14)$ & 74.57(68.24) & $110.40(80.00)$ & $\stackrel{<}{0.001}$ \\
\hline
\end{tabular}




\begin{tabular}{|c|c|c|c|c|}
\hline & $\begin{array}{l}\text { Overall } \\
\text { population }\end{array}$ & $\begin{array}{l}\text { D-dimers < } \\
1128 \mathrm{ng} / \mathrm{mL}\end{array}$ & $\begin{array}{l}\text { D-dimers } \geq \\
1128 \mathrm{ng} / \mathrm{mL}\end{array}$ & $\begin{array}{l}\mathrm{p}- \\
\text { value }\end{array}$ \\
\hline Fibrinogen, $\mathrm{g} / \mathrm{L}$, mean (SD) & $6.00(1.66)$ & $5.76(1.57)$ & $6.24(1.71)$ & $<.001$ \\
\hline $\begin{array}{l}\text { Ferritin, microg/L, mean } \\
\text { (SD) }\end{array}$ & $1063.80(1508.13)$ & $1000.28(1504.82)$ & $1121.25(1512.83)$ & 0.449 \\
\hline PT ratio (\%), mean (SD) & $85.47(18.16)$ & $87.40(18.67)$ & 83.36(17.37) & $\dot{0} 001$ \\
\hline aPTT ratio, mean (SD) & $1.15(0.31)$ & $1.15(0.32)$ & $1.15(0.30)$ & 0.864 \\
\hline \multicolumn{5}{|l|}{$\begin{array}{l}\text { Abnormalities on chest } \mathrm{CT} \text {, } \\
\mathrm{n}(\%)\end{array}$} \\
\hline $\begin{array}{l}\text { Parenchymal involvement } \\
\text { low or moderate }(<50 \%)\end{array}$ & $762(66.0)$ & $436(71.6)$ & $326(59.8)$ & $\begin{array}{l}< \\
0.001\end{array}$ \\
\hline $\begin{array}{l}\text { Parenchymal involvement } \\
\text { severe }(>50 \%)\end{array}$ & 201(17.4) & $80(13.1)$ & $121(22.2)$ & \\
\hline No chest CT-scan & 191(16.6) & $93(15.3)$ & $98(18.0)$ & \\
\hline \multicolumn{5}{|l|}{ Outcomes } \\
\hline Duration of stay, mean (SD) & $9.36(6.14)$ & $8.75(5.83)$ & $10.25(6.47)$ & 0.001 \\
\hline $\begin{array}{l}\text { Time from admission to in- } \\
\text { hospital death, mean (SD) }\end{array}$ & 15.22(10.29) & $16.6(7.82)$ & 13.7(9.19) & 0.001 \\
\hline In-hospital death, n(\%) & $121(10.5)$ & $35(5.7)$ & $86(15.8)$ & $\dot{0} 001$ \\
\hline
\end{tabular}

We also evaluated D-dimer level at admission in the subgroup of patients who developed VTE during hospitalization ( $n=127)$. In this subgroup, the optimum cutoff value for $D$-dimers at admission to predict in-hospital mortality was $1202 \mathrm{ng} / \mathrm{mL}$ using ROC curve (Fig. 1) with a sensitivity of $61 \%(95 \% \mathrm{Cl} 0.17-$ $0.92)$ and a specificity of $25.3 \%(95 \% \mathrm{Cl} 0.12-0.58)$, a positive predictive value of $5.8 \%$ ( $95 \% \mathrm{Cl} 0.01-$ $0.16)$ and a negative predictive value of $95.3 \%(95 \% \mathrm{Cl} 0.84-0.98)$. AUC for in-hospital mortality was $63.7 \%$ (95\% Cl: $0.37-0.90)$. This cutoff value at $1202 \mathrm{ng} / \mathrm{mL}$ did not significantly differ from that of the whole study population $(p=0.92)$.

\section{Increased D-dimer level at admission is an independent predictor of COVID-19 in-hospital mortality}

Kaplan-Meier survival curves for D-dimer level showed that level $\geq 1128 \mathrm{ng} / \mathrm{mL}$ at admission was a significant predictor of in hospital mortality ( $p<0.001$, Fig. $2 A)$. Statistical significance of separation 
between two groups was achieved at 9 days. As shown in Table 2, D-dimer level $\geq 1128 \mathrm{ng} / \mathrm{mL}$ was significantly associated with higher in-hospital mortality (OR 2.08, 95\% $\mathrm{Cl} 1.24-3.54, \mathrm{p}=0.006)$ in the logistic regression. In the same way Cox proportional hazard analysis showed that D-dimer level $\geq$ $1128 \mathrm{ng} / \mathrm{ml}$ at admission was also a significant determinant for worst prognosis (HR $2.1195 \% \mathrm{Cl} 1.31-$ $3.4, p<0.01$ ) after adjustment (Fig. 3). In the sensitivity analysis, the D-dimer level at admission ROC curve for in-hospital mortality in the subgroup of patient with VTE during hospitalization $(n=127)$ was similar (Fig. 1b). Moreover, when the analysis was restricted to patients without censored outcome $(n=$ 886) the level of association between D-dimer level $\geq 1128 \mathrm{ng} / \mathrm{mL}$ and in-hospital mortality remained similar with an OR of $1.88(95 \% \mathrm{Cl} 1.08-3.31, \mathrm{p}=0.02)$ and HR of $2.2(95 \% \mathrm{Cl} 1.25-3.3 ; \mathrm{p}<0.01)$ (Table 3). 
Table 2a

Association between D-dimers cutoff of $1128 \mathrm{ng} / \mathrm{mL}$ and in-hospital mortality using logistic regression. OR = Odds Ratio; $\mathrm{Cl}=$ Confident Interval; DOACs = Direct Oral Anticoagulants, VKA = Vitamin K antagonist, SD = Standard Deviation, CT = Computerized Tomography. *Venous thrombosis event included deep vein thrombosis and Pulmonary Embolism.

\begin{tabular}{|c|c|c|c|c|}
\hline & Alive & $\begin{array}{l}\text { in- } \\
\text { hospital } \\
\text { death }\end{array}$ & OR (univariable) & OR (multivariable) \\
\hline D-dimers > 1128 ng/mL & $\begin{array}{l}459 \\
(44.4)\end{array}$ & $86(71.1)$ & $\begin{array}{l}3.07(2.05-4.69, \mathrm{p} \\
<0.001)\end{array}$ & $\begin{array}{l}2.08(1.24-3.54, p \\
=0.006)\end{array}$ \\
\hline \multicolumn{5}{|l|}{ Age } \\
\hline$(50,60]$ & $\begin{array}{l}467 \\
(18.6)\end{array}$ & $15(4.2)$ & $\begin{array}{l}1.90(0.82-4.75, p \\
=0.149)\end{array}$ & $\begin{array}{l}0.96(0.28-3.19, \mathrm{p} \\
=0.943)\end{array}$ \\
\hline$(60,70]$ & $\begin{array}{l}577 \\
(23.0)\end{array}$ & $45(12.5)$ & $\begin{array}{l}4.60(2.27-10.63 \\
p<0.001)\end{array}$ & $\begin{array}{l}0.88(0.28-2.84, p \\
=0.824)\end{array}$ \\
\hline$(70,80]$ & $\begin{array}{l}498 \\
(19.8)\end{array}$ & $77(21.4)$ & $\begin{array}{l}9.12(4.63-20.70 \\
p<0.001)\end{array}$ & $\begin{array}{l}3.49(1.40-9.99, p \\
=0.011)\end{array}$ \\
\hline$(80,90]$ & $\begin{array}{l}361 \\
(14.4)\end{array}$ & $\begin{array}{l}135 \\
(37.5)\end{array}$ & $\begin{array}{l}22.06(11.38- \\
49.57, p<0.001)\end{array}$ & $\begin{array}{l}9.74(3.81-28.59 \\
p<0.001)\end{array}$ \\
\hline$(90,110]$ & $\begin{array}{l}138 \\
(5.5)\end{array}$ & $80(22.2)$ & $\begin{array}{l}34.20(17.12- \\
78.40, p<0.001)\end{array}$ & $\begin{array}{l}14.94(5.23- \\
47.60, p<0.001)\end{array}$ \\
\hline \multicolumn{5}{|l|}{ Cancer } \\
\hline Cancer in remission & $\begin{array}{l}183 \\
(7.3)\end{array}$ & $43(11.9)$ & $\begin{array}{l}1.87(1.30-2.64, p \\
=0.001)\end{array}$ & $\begin{array}{l}0.80(0.33-1.76, p \\
=0.598)\end{array}$ \\
\hline Active cancer & $\begin{array}{l}146 \\
(5.8)\end{array}$ & $43(11.9)$ & $\begin{array}{l}2.34(1.61-3.34, p \\
<0.001)\end{array}$ & $\begin{array}{l}1.84(0.77-4.11, p \\
=0.149)\end{array}$ \\
\hline High blood pressure & $\begin{array}{l}1191 \\
(47.6)\end{array}$ & $\begin{array}{l}262 \\
(73.0)\end{array}$ & $\begin{array}{l}2.97(2.33-3.81, p \\
<0.001)\end{array}$ & $\begin{array}{l}0.97(0.56-1.69, p \\
=0.918)\end{array}$ \\
\hline $\begin{array}{l}\text { Oral anticoagulation (DOACs or } \\
\text { VKA) }\end{array}$ & $\begin{array}{l}298 \\
(12.0)\end{array}$ & $84(23.5)$ & $\begin{array}{l}2.26(1.72-2.96, \mathrm{p} \\
<0.001)\end{array}$ & $\begin{array}{l}1.08(0.53-2.10, p \\
=0.818)\end{array}$ \\
\hline $\begin{array}{l}\text { Plasma creatinine level - } \\
\mu \mathrm{mol} / \mathrm{L} \text {, mean (SD) }\end{array}$ & $\begin{array}{l}92.3 \\
(86.4)\end{array}$ & $\begin{array}{l}139.6 \\
(137.5)\end{array}$ & $\begin{array}{l}1.00(1.00-1.00, \mathrm{p} \\
<0.001)\end{array}$ & $\begin{array}{l}1.00(1.00-1.00, p \\
=0.001)\end{array}$ \\
\hline $\begin{array}{l}\text { Parenchymal opacification in } \\
\text { chest CT-scan }>50 \%\end{array}$ & $\begin{array}{l}356 \\
(17.8)\end{array}$ & $74(30.0)$ & $\begin{array}{l}1.98(1.46-2.64, \mathrm{p} \\
<0.001)\end{array}$ & $\begin{array}{l}2.00(1.16-3.42, p \\
=0.012)\end{array}$ \\
\hline Venous thrombosis event* & $\begin{array}{l}116 \\
(4.6)\end{array}$ & $11(3.0)$ & $\begin{array}{l}0.65(0.33-1.17, p \\
=0.180)\end{array}$ & $\begin{array}{l}0.72(0.20-1.98, p \\
=0.562)\end{array}$ \\
\hline
\end{tabular}




\section{Table $2 b$}

b. Association between D-dimers cutoff of $1128 \mathrm{ng} / \mathrm{mL}$ and in-hospital mortality using logistic regression in the selected population of patient without censored outcome. OR = Odds Ratio; $\mathrm{Cl}$ = Confident Interval;

DOACs = Direct Oral Anticoagulants, VKA = Vitamin K antagonist, SD = Standard Deviation, CT =

Computerized Tomography. *Venous thrombosis event included deep vein thrombosis and Pulmonary

Embolism.

\begin{tabular}{|c|c|c|c|c|}
\hline & Alive & $\begin{array}{l}\text { in- } \\
\text { hospital } \\
\text { death }\end{array}$ & OR (univariable) & OR (multivariable) \\
\hline D-dimers $>1128 \mathrm{ng} / \mathrm{mL}$ & $\begin{array}{l}313 \\
(40.9)\end{array}$ & $86(71.1)$ & $\begin{array}{l}3.55(2.35-5.45, \mathrm{p} \\
<0.001)\end{array}$ & $\begin{array}{l}1.88(1.08-3.30, p \\
=0.026)\end{array}$ \\
\hline \multicolumn{5}{|l|}{ Age } \\
\hline$(50,60]$ & $\begin{array}{l}157 \\
(20.5)\end{array}$ & $7(5.8)$ & $\begin{array}{l}1.23(0.41-3.66, p \\
=0.705)\end{array}$ & $\begin{array}{l}1.07(0.32-3.61, p \\
=0.909)\end{array}$ \\
\hline$(60,70]$ & $\begin{array}{l}168 \\
(22.0)\end{array}$ & $19(15.8)$ & $\begin{array}{l}3.12(1.33-8.15, p \\
=0.012)\end{array}$ & $\begin{array}{l}1.03(0.32-3.41, p \\
=0.953)\end{array}$ \\
\hline$(70,80]$ & $\begin{array}{l}133 \\
(17.4)\end{array}$ & $25(20.8)$ & $\begin{array}{l}5.18(2.29-13.30 \\
p<0.001)\end{array}$ & $\begin{array}{l}4.47(1.73-13.17 \\
p=0.003)\end{array}$ \\
\hline$(80,90]$ & $\begin{array}{l}86 \\
(11.3)\end{array}$ & $40(33.3)$ & $\begin{array}{l}12.82(5.86-32.33 \\
p<0.001)\end{array}$ & $\begin{array}{l}9.70(3.66-29.38 \\
\mathrm{p}<0.001)\end{array}$ \\
\hline$(90,110]$ & $\begin{array}{l}27 \\
(3.5)\end{array}$ & $22(18.3)$ & $\begin{array}{l}22.47(9.17-61.57 \\
p<0.001)\end{array}$ & $\begin{array}{l}18.04(5.78-62.28 \\
p<0.001)\end{array}$ \\
\hline \multicolumn{5}{|l|}{ Cancer } \\
\hline Cancer in remission & $\begin{array}{l}67 \\
(8.8)\end{array}$ & $12(9.9)$ & $\begin{array}{l}1.25(0.62-2.31, \mathrm{p} \\
=0.505)\end{array}$ & $\begin{array}{l}0.80(0.32-1.84, p \\
=0.622)\end{array}$ \\
\hline Active cancer & $\begin{array}{l}36 \\
(4.7)\end{array}$ & $14(11.6)$ & $\begin{array}{l}2.71(1.37-5.11, p \\
=0.003)\end{array}$ & $\begin{array}{l}2.80(1.06-6.99, p \\
=0.031)\end{array}$ \\
\hline High blood pressure & $\begin{array}{l}333 \\
(43.8)\end{array}$ & $80(66.7)$ & $\begin{array}{l}2.56(1.72-3.88, \mathrm{p} \\
<0.001)\end{array}$ & $\begin{array}{l}0.92(0.51-1.64, p \\
=0.767)\end{array}$ \\
\hline $\begin{array}{l}\text { Oral anticoagulation (DOA or } \\
\text { VKA) }\end{array}$ & $\begin{array}{l}64 \\
(8.4)\end{array}$ & $23(19.0)$ & $\begin{array}{l}2.56(1.50-4.26, \mathrm{p} \\
<0.001)\end{array}$ & $\begin{array}{l}1.46(0.68-3.01, p \\
=0.320)\end{array}$ \\
\hline $\begin{array}{l}\text { Plasma creatinine level - } \mu \mathrm{mol} / \mathrm{L} \text {, } \\
\text { mean (SD) }\end{array}$ & $\begin{array}{l}86.6 \\
(63.0)\end{array}$ & $\begin{array}{l}139.2 \\
(135.4)\end{array}$ & $\begin{array}{l}1.01(1.00-1.01, p \\
<0.001)\end{array}$ & $\begin{array}{l}1.01(1.00-1.01, p \\
<0.001)\end{array}$ \\
\hline $\begin{array}{l}\text { Parenchymal opacification in } \\
\text { chest CT-scan }>50 \%\end{array}$ & $\begin{array}{l}98 \\
(15.0)\end{array}$ & $30(32.6)$ & $\begin{array}{l}2.74(1.67-4.42, \mathrm{p} \\
<0.001)\end{array}$ & $\begin{array}{l}3.01(1.64-5.49, p \\
<0.001)\end{array}$ \\
\hline Venous thrombosis event* & $\begin{array}{l}43 \\
(5.6)\end{array}$ & $5(4.1)$ & $\begin{array}{l}0.72(0.25-1.70, p \\
=0.503)\end{array}$ & $\begin{array}{l}1.05(0.29-3.07, p \\
=0.929)\end{array}$ \\
\hline
\end{tabular}


Table 3

Association between D-dimer cutoff of $1128 \mathrm{ng} / \mathrm{mL}$ and in-hospital mortality using logistic regression in the selected population of patients without censored outcome.

\begin{tabular}{|c|c|c|c|c|}
\hline & Alive & $\begin{array}{l}\text { in- } \\
\text { hospital } \\
\text { death }\end{array}$ & OR (univariable) & OR (multivariable) \\
\hline D-dimers > $1128 \mathrm{ng} / \mathrm{mL}$ & $\begin{array}{l}459 \\
(44.4)\end{array}$ & $86(71.1)$ & $\begin{array}{l}3.07(2.05-4.69, p \\
<0.001)\end{array}$ & $\begin{array}{l}2.08(1.24-3.54, p \\
=0.006)\end{array}$ \\
\hline \multicolumn{5}{|l|}{ Age } \\
\hline$(50,60]$ & $\begin{array}{l}467 \\
(18.6)\end{array}$ & $15(4.2)$ & $\begin{array}{l}1.90(0.82-4.75, \mathrm{p} \\
=0.149)\end{array}$ & $\begin{array}{l}0.96(0.28-3.19, p \\
=0.943)\end{array}$ \\
\hline$(60,70]$ & $\begin{array}{l}577 \\
(23.0)\end{array}$ & $45(12.5)$ & $\begin{array}{l}4.60(2.27-10.63 \\
p<0.001)\end{array}$ & $\begin{array}{l}0.88(0.28-2.84, p \\
=0.824)\end{array}$ \\
\hline$(70,80]$ & $\begin{array}{l}498 \\
(19.8)\end{array}$ & $77(21.4)$ & $\begin{array}{l}9.12(4.63-20.70 \\
p<0.001)\end{array}$ & $\begin{array}{l}3.49(1.40-9.99, p \\
=0.011)\end{array}$ \\
\hline$(80,90]$ & $\begin{array}{l}361 \\
(14.4)\end{array}$ & $\begin{array}{l}135 \\
(37.5)\end{array}$ & $\begin{array}{l}22.06(11.38- \\
49.57, p<0.001)\end{array}$ & $\begin{array}{l}9.74(3.81-28.59 \\
p<0.001)\end{array}$ \\
\hline$(90,110]$ & $\begin{array}{l}138 \\
(5.5)\end{array}$ & $80(22.2)$ & $\begin{array}{l}34.20(17.12- \\
78.40, p<0.001)\end{array}$ & $\begin{array}{l}14.94(5.23-47.60, \\
p<0.001)\end{array}$ \\
\hline Cancer in remission & $\begin{array}{l}183 \\
(7.3)\end{array}$ & $43(11.9)$ & $\begin{array}{l}1.87(1.30-2.64, p \\
=0.001)\end{array}$ & $\begin{array}{l}0.80(0.33-1.76, p \\
=0.598)\end{array}$ \\
\hline Active cancer & $\begin{array}{l}146 \\
(5.8)\end{array}$ & $43(11.9)$ & $\begin{array}{l}2.34(1.61-3.34, p \\
<0.001)\end{array}$ & $\begin{array}{l}1.84(0.77-4.11, \mathrm{p} \\
=0.149)\end{array}$ \\
\hline High blood pressure & $\begin{array}{l}1191 \\
(47.6)\end{array}$ & $\begin{array}{l}262 \\
(73.0)\end{array}$ & $\begin{array}{l}2.97(2.33-3.81, p \\
<0.001)\end{array}$ & $\begin{array}{l}0.97(0.56-1.69, p \\
=0.918)\end{array}$ \\
\hline $\begin{array}{l}\text { Oral anticoagulation (DOACs or } \\
\text { VKA) }\end{array}$ & $\begin{array}{l}298 \\
(12.0)\end{array}$ & $84(23.5)$ & $\begin{array}{l}2.26(1.72-2.96, p \\
<0.001)\end{array}$ & $\begin{array}{l}1.08(0.53-2.10, p \\
=0.818)\end{array}$ \\
\hline $\begin{array}{l}\text { Plasma creatinine level - } \\
\mu \mathrm{mol} / \mathrm{L} \text {, mean (SD) }\end{array}$ & $\begin{array}{l}92.3 \\
(86.4)\end{array}$ & $\begin{array}{l}139.6 \\
(137.5)\end{array}$ & $\begin{array}{l}1.00(1.00-1.00, p \\
<0.001)\end{array}$ & $\begin{array}{l}1.00(1.00-1.00, \mathrm{p} \\
=0.001)\end{array}$ \\
\hline $\begin{array}{l}\text { Parenchymal opacification in } \\
\text { chest CT-scan }>50 \%\end{array}$ & $\begin{array}{l}356 \\
(17.8)\end{array}$ & $74(30.0)$ & $\begin{array}{l}1.98(1.46-2.64, p \\
<0.001)\end{array}$ & $\begin{array}{l}2.00(1.16-3.42, p \\
=0.012)\end{array}$ \\
\hline Venous thrombosis event & $\begin{array}{l}116 \\
(4.6)\end{array}$ & $11(3.0)$ & $\begin{array}{l}0.65(0.33-1.17, p \\
=0.180)\end{array}$ & $\begin{array}{l}0.72(0.20-1.98, p \\
=0.562)\end{array}$ \\
\hline $\begin{array}{l}\mathrm{OR}=\text { Odds Ratio; } \mathrm{Cl}=\text { Confidenc } \\
\text { antagonist, } \mathrm{SD}=\mathrm{Standard} \text { Devi } \\
\text { included deep vein thrombosis }\end{array}$ & $\begin{array}{ll}\mathrm{CT} \\
\mathrm{Clm}\end{array}$ & $=\mathrm{Di}$ & al Anticoagulan & $\begin{array}{l}=\text { Vitamin } \mathrm{K} \\
\text { ombosis event }\end{array}$ \\
\hline
\end{tabular}




\section{Discussion}

The main finding of this retrospective study is that D-dimer level at admission above $1128 \mathrm{ng} / \mathrm{mL}$ is an independent predictor of in-hospital mortality for COVID-19 patients. This multicenter French study of patients hospitalized for COVID-19 is the current largest non-monocentric study to date for hospitalized patients in medical ward to provide evidence that initial D-dimer levels could be a valuable tool to predict further in-hospital mortality. Moreover, to the best of our knowledge, we show for the first time that VTE occurrence during hospitalization did not interfere with the predictive value of D-dimers for in-hospital mortality.

High D-dimer level has been largely reported to be one of the most common laboratory findings reported in COVID-19 patients at hospital admission. We previously demonstrated that D-dimer measurement at admission is a discriminant factor during COVID-19 suspicion. Indeed, adding a D-dimer cut-off beyond $500 \mathrm{ng} / \mathrm{mL}$ to female gender and absence of pneumonia at CT scan could exclude COVID-19 diagnosis with a high sensitivity and specificity (4). Moreover, we and others previously showed that D-dimer level at admission was higher in patients needing ICU referral compared to those who did not require it $(5,20)$. Moreover, several reports have described that increased D-dimer levels were related to in-hospital mortality $(14,21-23)$. Only one study provided a well evaluated cutoff for D-dimers (15) at $2000 \mathrm{ng} / \mathrm{mL}$ for relation with in hospital mortality in 343 patients. However, this study did not specify if patients were hospitalized in medical ward, in ICU or if patients were directly hospitalized in ICU, making proper and accurate use of this cut-off difficult for clinicians. Our study only includedCOVID-19 patients admitted in medical ward. Some of them were secondary referred to ICU but no one was directly hospitalized in ICU. Our results propose COVID-19-increased D-dimer level as a clear consequence of respiratory disease through the development of capillary microthrombosis, as observed in post-mortem studies $(3,11)$ and attributed to a vascular thickening or vascular congestion (24). Thus, in COVID-19, hypothesis of microthrombosis is proposed in lung but also in kidney since the elevation of serum creatinine was associated with higher levels of D-dimers (> $500 \mathrm{ng} / \mathrm{mL}$ ) (25). The SARS-CoV-2 receptor (ACE2) is strongly expressed in endothelial cells (26). Infection of endothelial cells could therefore induce endothelial lesions triggering massive activation of coagulation and diffuse microthrombotic process impairing renal function and respiratory gas exchanges. We previously described increased numbers of circulating endothelial cells in COVID-19 patients (4) and an association between circulating biomarkers of endothelial activation in COVID-19 and ICU admission (5). Angiopoietin-2 was also inversely correlated to respiratory system compliance in this study, paving the way of relationship between endothelial dysfunction and pulmonary disease severity. Integrity of endothelial cells allows providing an antithrombotic environment that is reversed during COVID-19 upon the burst of inflammation related to IL-6. Therefore, SARS-CoV-2 infection induces a disruption of endothelial thrombo-protective barrier that lead to this coagulopathy and increased D-dimers. Since in the present cohort, patients were in the same step of disease according to same time to onset symptoms of disease, endothelial induced coagulopathy reflected by D-dimers could be a consequence of viral loading phase and severity of viral infection. 
Importance in viral loading hypothesis needs to be confirmed with association between D-dimers and viremia quantified with sensitive tests.

Major confounding factor for D-dimers increase could be macrothrombosis since high incidence of VTE (PE or DVT) $(7,9,27)$ have been described in COVID-19. In clinical practice, D-dimer measurements have been used only to exclude VTE. Indeed, no such D-dimer-based strategy has been described during COVID-19-associated coagulopathy with patients with a high level of D-dimers. Even if increased D-dimer levels at admission have been associated with VTE during follow-up in COVID-19 patients (28), no threshold is currently available to diagnose VTE. Furthermore, the International Society of thrombosis and Haemostasis (ISTH) does not recommend routine screening for VTE based on elevated D-dimer levels in COVID-19 patients (29). We demonstrate here that $1128 \mathrm{ng} / \mathrm{mL} \mathrm{D-dimer} \mathrm{cut-off} \mathrm{at} \mathrm{admission} \mathrm{is}$ independently correlated to in-hospital mortality regardless VTE occurrence during hospitalization. Ddimers might be used to monitor COVID-19 worsening. Indeed, previous studies have observed that progressive increase of D-dimers was observed in non-survivors of COVID-19 (1). Microthrombosis generating D-dimers allow to makes prognosis in COVID-19 outcome and also opens the way of D-dimer monitoring to guide whether anticoagulation therapy should be initiated in COVID-19. D-dimer monitoring has been described in a randomized clinical trial in patients with mechanical valve replacement as a good tool to guide anticoagulation intensity in patients receiving warfarin therapy (30) but also to determine the duration of oral anticoagulation in patients with VTE (31). Thus, our results suggest that therapeutic anticoagulation could be initiated in COVID-19 patients with high D-dimer level at admission in contrast to patients with $\mathrm{D}$-dimers beyond this cut-off that should only benefit from prophylactic anticoagulation. Moreover, D-dimer-based strategy to guide anticoagulation regimen needs to be evaluated in prospective randomized clinical trials.

Our study has several limitations. First, in this multicentric study, we could not identify the manufacturer or type of D-dimer assay used for all tested D-dimers as suggested by ISTH (32) Second, we do not have the delay from COVID-19 admission to VTE onset during hospitalization. Third, serial D-dimer monitoring has been suggested by ISTH (32) as helpful in determining prognosis in COVID-19 patients. Indeed, a peak of D-dimers has been found associated with VTE in $\operatorname{COVID-19}(33,34)$ but in the present study, we only assessed D-dimers at admission. However, since VTE occurrence did not to modify in-hospital mortality in the present study, this lack of continuous monitoring of D-dimers is unlikely to modify results.

\section{Conclusion}

In conclusion, this multicentric retrospective study suggests that D-dimer level at admission could be a valuable biomarker to predict mortality related to COVID-19, independently of VTE occurrence during hospitalization. The determined cut-off at $1128 \mathrm{ng} / \mathrm{mL}$ could be a valuable tool to guide anticoagulation intensity in COVID-19 patients. Further prospective studies are necessary to confirm this threshold of Ddimers reflecting COVID-19 worsening.

\section{Abbreviations}


$95 \% \mathrm{Cl}=95 \%$ Confident interval

ACE2 = Angiotensin-converting enzyme 2SARS-CoV-2 receptor

CCF $=$ Critical COVID-19 France

COVID-19 = Coronavirus disease 2019

$\mathrm{CT}=$ Computed tomography

DVT = Deep Veneous Thrombosis

$\mathrm{ICU}=$ Intensive care unit

ISTH = International Society of thrombosis and Haemostasis

$\mathrm{OR}=$ Odds ratio

$\mathrm{PE}=$ Pulmonary embolism

$\mathrm{PH}=$ Proportional hazard

PT = Prothrombin

ROC $=$ Receiver operating characteristic

SARS-CoV-2 = Severe acute respiratory syndrome coronavirus 2

$\mathrm{SD}=$ Standard deviation

VTE $=$ Venous thromboembolism

\section{Declarations}

\section{Ethics approval and consent to participate}

The CCF study was declared and authorized by the French data protection committee (authorization no. 2207326v0) and conducted in accordance with the ethical standards established in the 1964 Declaration of Helsinki and its later amendments (NCT04344327).

\section{Consent for publication}

Not applicable 


\section{Availability of data and material}

Please contact author for data requests

\section{Competing interests}

Richard Chocron, Ariel Cohen and David Smadja acknowledge the following without any relation with the current manuscript. Richard Chocron received Consultant fees from the Aspen Company. Ariel Cohen received research grant from RESICARD (research nurses) and consultant and lecture fees from Amgen, AstraZeneca, Bayer Pharma, Alliance BMS-Pfizer, Novartis, and Sanofi-Aventis. David Smadja received consultant, lecture fees or travel awards from Aspen, Bayer, Carmat, Alliance BMS-Pfizer, Léo Pharma and Boehringer-Ingelheim. The other authors have nothing to disclose.

\section{Funding}

This research did not receive any specific grant from funding agencies in the public, commercial, or notfor-profit sectors.

\section{Authors' contributions}

All the undersigning authors have substantially contributed to the paper. David Smadja and Richard Chocron designed the present study and wrote the manuscript. Richard Chocron performed statistical analyses. Ariel Cohen and Guillaume Bonnet designed the trial.

All authors declare that the submitted work is original and has not been published before (neither in English nor in any other language) and that the work is not under consideration for publication elsewhere.

\section{Acknowledgements}

Not applicable

\section{References}

1. Zhou F, Yu T, Du R, Fan G, Liu Y, Liu Z, Xiang J, Wang Y, Song B, Gu X, Guan L, Wei Y, Li H, Wu X, Xu J, Tu S, Zhang Y, Chen H, Cao B. Clinical course and risk factors for mortality of adult inpatients with COVID-19 in Wuhan, China: a retrospective cohort study. Lancet 2020.

2. Debuc B, Smadja DM. Is COVID-19 a New Hematologic Disease? Stem Cell Rev Rep 2020; In press.

3. Ackermann M, Verleden SE, Kuehnel M, Haverich A, Welte T, Laenger F, Vanstapel A, Werlein C, Stark H, Tzankov A, Li WW, Li VW, Mentzer SJ, Jonigk D. Pulmonary Vascular Endothelialitis, Thrombosis, 
and Angiogenesis in Covid-19. N Engl J Med 2020.

4. Khider L, Gendron N, Goudot G, Chocron R, Hauw-Berlemont C, Cheng C, Rivet N, Pere H, Roffe A, Clerc S, Lebeaux D, Debuc B, Veyer D, Rance B, Gaussem P, Bertil S, Badoual C, Juvin P, Planquette B, Messas E, et al. Curative anticoagulation prevents endothelial lesion in COVID-19 patients. J Thromb Haemost 2020; In press.

5. Smadja DM, Guerin CL, Chocron R, Yatim N, Boussier J, Gendron N, Khider L, Hadjadj J, Goudot G, Debuc B, Juvin P, Hauw-Berlemont C, Augy JL, Peron N, Messas E, Planquette B, Sanchez O, Charbit B, Gaussem P, Duffy D, et al. Angiopoietin-2 as a marker of endothelial activation is a good predictor factor for intensive care unit admission of COVID-19 patients. Angiogenesis 2020; In press.

6. Varga Z, Flammer AJ, Steiger P, Haberecker M, Andermatt R, Zinkernagel AS, Mehra MR, Schuepbach RA, Ruschitzka F, Moch H. Endothelial cell infection and endotheliitis in COVID-19. Lancet 2020.

7. Poissy J, Goutay J, Caplan M, Parmentier E, Duburcq T, Lassalle F, Jeanpierre E, Rauch A, Labreuche J, Susen S. Pulmonary Embolism in COVID-19 Patients: Awareness of an Increased Prevalence. Circulation 2020.

8. Voicu S, Bonnin P, Stepanian A, Chousterman BG, Le Gall A, Malissin I, Deye N, Siguret V, Mebazaa A, Megarbane $B$. High prevalence of deep vein thrombosis in mechanically ventilated COVID-19 patients. J Am Coll Cardiol 2020.

9. Fauvel C, Weizman O, Trimaille A, Mika D, Pommier T, Pace N, Douair A, Barbin E, Fraix A, Bouchot O, Benmansour O, Godeau G, Mecheri Y, Lebourdon R, Yvorel C, Massin M, Leblon T, Chabbi C, Cugney E, Benabou L, et al. Pulmonary embolism in COVID-19 patients: a French multicentre cohort study. Eur Heart J 2020.

10. Fox S, Akmatbekov A, Harbert J, Li G, Brown G, Vander Heide R. Pulmonary and Cardiac Pathology in Covid-19: The First Autopsy Series from New Orleans. medRxiv preprint 2020.

11. Zhang H, Zhou P, Wei Y, Yue H, Wang Y, Hu M, Zhang S, Cao T, Yang C, Li M, Guo G, Chen X, Chen Y, Lei M, Liu H, Zhao J, Peng P, Wang CY, Du R. Histopathologic Changes and SARS-CoV-2 Immunostaining in the Lung of a Patient With COVID-19. Ann Intern Med 2020.

12. Diehl JL, Peron N, Chocron R, Debuc B, Guerot E, Hauw-Berlemont C, Hermann B, Augy JL, Younan R, Novara A, Langlais J, Khider L, Gendron N, Goudot G, Fagon JY, Smadja DM. Respiratory mechanics and gas exchanges in the early course of COVID-19 ARDS: a hypothesis-generating study. Ann Intensive Care 2020.

13. Connors JM, Levy JH. COVID-19 and its implications for thrombosis and anticoagulation. Blood. 2020;135:2033-40.

14. Tang N, Li D, Wang X, Sun Z. Abnormal Coagulation parameters are associated with poor prognosis in patients with novel coronavirus pneumonia. J Thromb Haemost 2020.

15. Zhang L, Yan X, Fan Q, Liu H, Liu X, Liu Z, Zhang Z. D-dimer levels on admission to predict in-hospital mortality in patients with Covid-19. J Thromb Haemost. 2020;18:1324-9.

16. Revel MP, Parkar AP, Prosch H, Silva M, Sverzellati N, Gleeson F, Brady A. COVID-19 patients and the radiology department - advice from the European Society of Radiology (ESR) and the European 
Society of Thoracic Imaging (ESTI). Eur Radiol 2020.

17. Bewick V, Cheek L, Ball J. Statistics review 10: further nonparametric methods. Crit Care. 2004;8:196-9.

18. Reichenheim ME, Coutinho ES. Measures and models for causal inference in cross-sectional studies: arguments for the appropriateness of the prevalence odds ratio and related logistic regression. BMC Med Res Methodol. 2010;10:66.

19. Sedgwick P. Bias in observational study designs: cross sectional studies. Bmj. 2015;350:h1286.

20. Huang C, Wang Y, Li X, Ren L, Zhao J, Hu Y, Zhang L, Fan G, Xu J, Gu X, Cheng Z, Yu T, Xia J, Wei Y, Wu W, Xie X, Yin W, Li H, Liu M, Xiao Y, et al. Clinical features of patients infected with 2019 novel coronavirus in Wuhan, China. Lancet. 2020;395:497-506.

21. Guan WJ, Ni ZY, Hu Y, Liang WH, Ou CQ, He JX, Liu L, Shan H, Lei CL, Hui DSC, Du B, Li LJ, Zeng G, Yuen KY, Chen RC, Tang CL, Wang T, Chen PY, Xiang J, Li SY, et al. Clinical Characteristics of Coronavirus Disease 2019 in China. N Engl J Med 2020.

22. Chen N, Zhou M, Dong X, Qu J, Gong F, Han Y, Qiu Y, Wang J, Liu Y, Wei Y, Xia J, Yu T, Zhang X, Zhang L. Epidemiological and clinical characteristics of 99 cases of 2019 novel coronavirus pneumonia in Wuhan, China: a descriptive study. Lancet. 2020;395:507-13.

23. Fei Y, Tang N, Liu H, Cao W. Coagulation dysfunction: A hallmark in COVID-19. Arch Pathol Lab Med 2020.

24. Price LC, McCabe C, Garfield B, Wort SJ. Thrombosis and COVID-19 pneumonia: the clot thickens! Eur Respir J 2020.

25. Cheng Y, Luo R, Wang K, Zhang M, Wang M, Dong L, Li J, Yao Y, Ge S, Xu G. Kidney disease is associated with in-hospital death of patients with COVID-19. Kidney International 2020; In press.

26. Ferrario CM, Jessup J, Chappell MC, Averill DB, Brosnihan KB, Tallant EA, Diz DI, Gallagher PE. Effect of angiotensin-converting enzyme inhibition and angiotensin II receptor blockers on cardiac angiotensin-converting enzyme 2. Circulation. 2005;111:2605-10.

27. Helms J, Severac F, Merdji H, Angles-Cano E, Meziani F. Prothrombotic phenotype in COVID-19 severe patients. Intensive Care Med 2020.

28. Middeldorp S, Coppens M, van Haaps TF, Foppen M, Vlaar AP, Muller MCA, Bouman CCS, Beenen LFM, Kootte RS, Heijmans J, Smits LP, Bonta PI, van Es N. Incidence of venous thromboembolism in hospitalized patients with COVID-19. J Thromb Haemost 2020.

29. Spyropoulos AC, Levy JH, Ageno W, Connors JM, Hunt BJ, Iba T, Levi M, Samama CM, Thachil J, Giannis D, Douketis JD. Scientific and Standardization Committee Communication: Clinical Guidance on the Diagnosis, Prevention and Treatment of Venous Thromboembolism in Hospitalized Patients with COVID-19. J Thromb Haemost 2020.

30. Zhang L, Zheng X, Long Y, Wu M, Chen Y, Yang J, Liu Z, Zhang Z. D-dimer to guide the intensity of anticoagulation in Chinese patients after mechanical heart valve replacement: a randomized controlled trial. J Thromb Haemost. 2017;15:1934-41. 
31. Zhang L, Long Y, Xiao H, Yang J, Toulon P, Zhang Z. Use of D-dimer in oral anticoagulation therapy. Int J Lab Hematol 2018.

32. Thachil J, Tang N, Gando S, Falanga A, Cattaneo M, Levi M, Clark C, Iba T. ISTH interim guidance on recognition and management of coagulopathy in COVID-19. J Thromb Haemost 2020; In press.

33. Faggiano P, Bonelli A, Paris S, Milesi G, Bisegna S, Bernardi N, Curnis A, Agricola E, Maroldi R. Acute pulmonary embolism in COVID-19 disease: Preliminary report on seven patients. Int J Cardiol. 2020;313:129-31.

34. Maatman TK, Jalali F, Feizpour C, Douglas A 2nd, McGuire SP, Kinnaman G, Hartwell JL, Maatman BT, Kreutz RP, Kapoor R, Rahman O, Zyromski NJ, Meagher AD. Routine Venous Thromboembolism Prophylaxis May Be Inadequate in the Hypercoagulable State of Severe Coronavirus Disease 2019. Crit Care Med 2020.

\section{Figures}

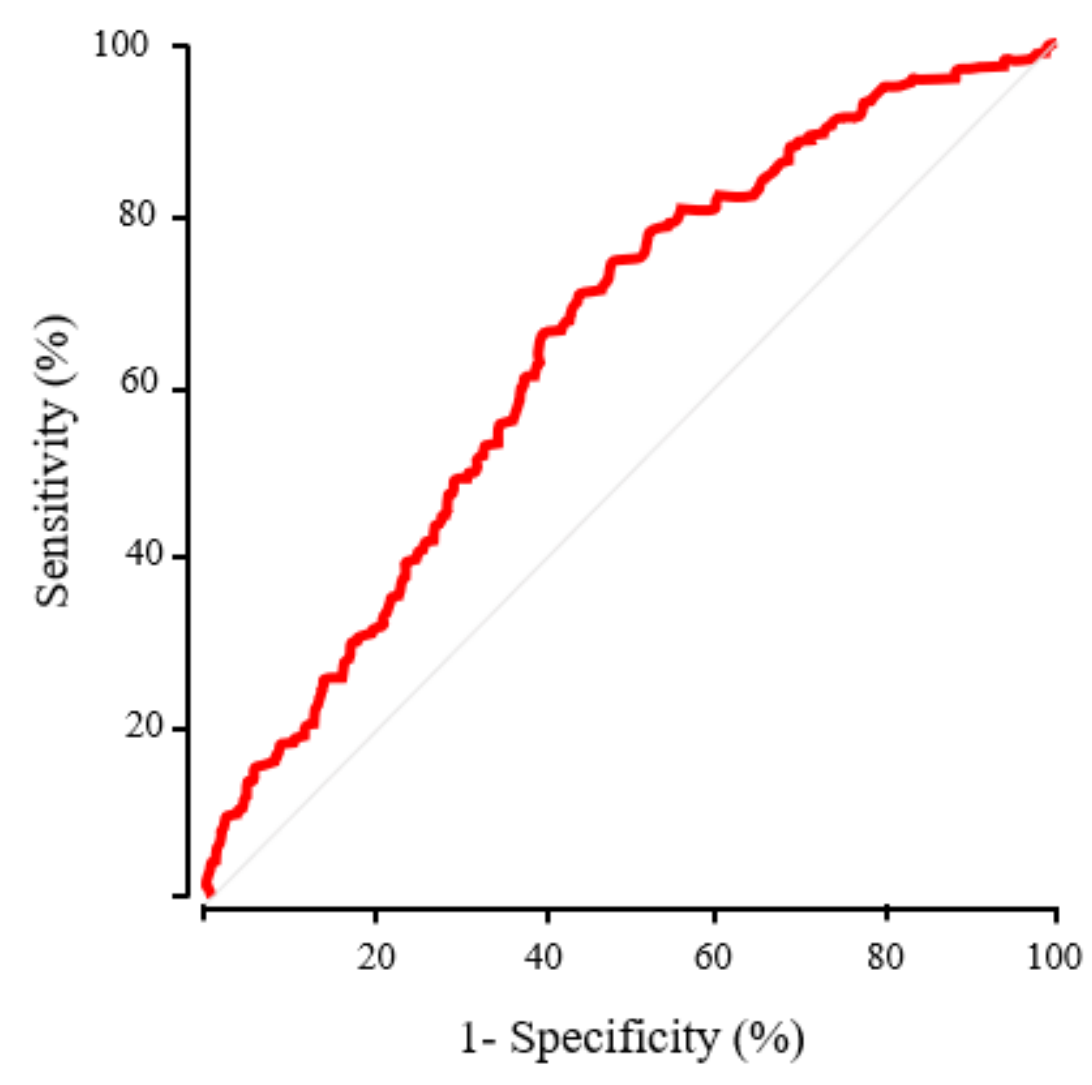

\begin{tabular}{|c|c|c|}
\hline & $\begin{array}{c}\text { D-dimers } \\
\text { Cut-off of } \mathbf{~ 1 1 2 8} \\
\mathbf{n g} / \mathbf{m l}\end{array}$ & CI 95\% \\
\hline Sensitivity & $\mathbf{7 1 . 1 \%}$ & $0.62-0.78$ \\
\hline Specificity & $\mathbf{5 5 . 6 \%}$ & $0.52-0.58$ \\
\hline PPV & $\mathbf{1 5 . 8} \%$ & $0.13-0.19$ \\
\hline NPV & $\mathbf{9 4 . 3} \%$ & $0.92-0.96$ \\
\hline
\end{tabular}


Figure 1

D-dimers level at admission receiver operating characteristic (ROC) curve for in-hospital mortality. (ng/ml). AUC $=64.9 \%$ (60\%-69.7\%). The D-dimers level at admission of $>1128 \mathrm{ng} / \mathrm{mL}$ represents optimal threshold using the Youden's $\mathrm{J}$ statistic. PPV= Positive predictive value; NPV= Negative predictive value; $\mathrm{Cl}$ 95\% $=95 \%$ Confidence interval.

D-dimer $>1128$

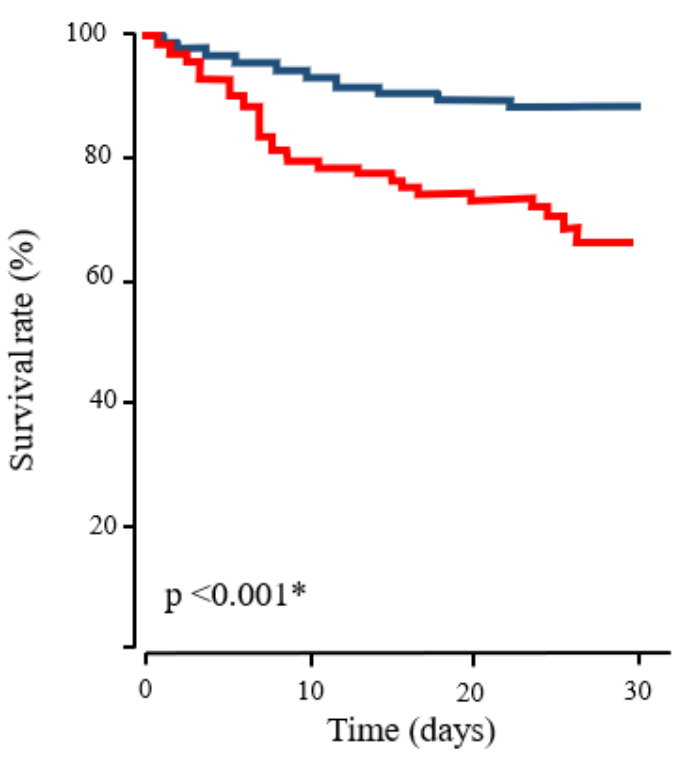

D-dimer $>1128$

dimer $>1128$

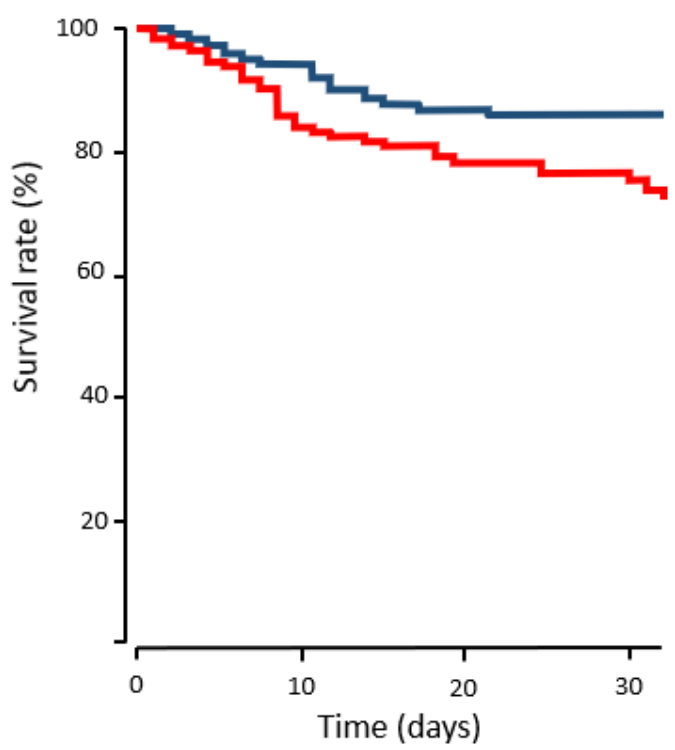

\begin{tabular}{|c|c|c|c|c|}
\hline \multicolumn{5}{|c|}{ Risk Table } \\
\hline & Days 0 & Days 10 & Days 20 & Days 30 \\
\hline $\begin{array}{c}\text { D-dimer } \\
<1128 \mathrm{ng} / \mathrm{mL}\end{array}$ & 539 & 388 & 237 & 68 \\
\hline $\begin{array}{c}\mathrm{D}-\text { dimer } \\
>1128 \mathrm{ng} / \mathrm{mL}\end{array}$ & 484 & 272 & 166 & 41 \\
\hline
\end{tabular}

A

B

\section{Figure 2}

a. Kaplan-Meier survival curves, illustrating the prognostic impact of the D-dimers threshold (1128 $\mathrm{ng} / \mathrm{mL}$ ) at admission. *Using the log-rank test. b. Adjusted Kaplan Meier Survival Curves for Cox Proportional Hazards (PH) Model that included age, history of malignancy, history of high blood pressure, the use of oral anticoagulation prior COVID-19, the level of plasma creatinine, abnormalities on chest CT ( $>$ or $>50 \%$ of parenchymental) and the occurrence of venous thrombosis event . Adjusted Survival Curves show how D-dimers threshold at admission of $1128 \mathrm{ng} / \mathrm{mL}$ influenced survival estimated from the Cox $\mathrm{PH}$ model. 
D-dimers $>1128 \mathrm{ng} / \mathrm{mL}$

Age

$(50,60]$

$(60,70]$

$(70,80]$

$(80,90]$

$(90,110]$

Cancer

Cancer in remission

Active cancer

High blood pressure

Oral anticoagulation (DOACs or VKA)

Plasma creatinine level - $\mu \mathrm{mol} / \mathrm{L}$, mean (SD)

Parenchymal opacification in chest CT-scan $>50 \%$

Venous thrombosis event*

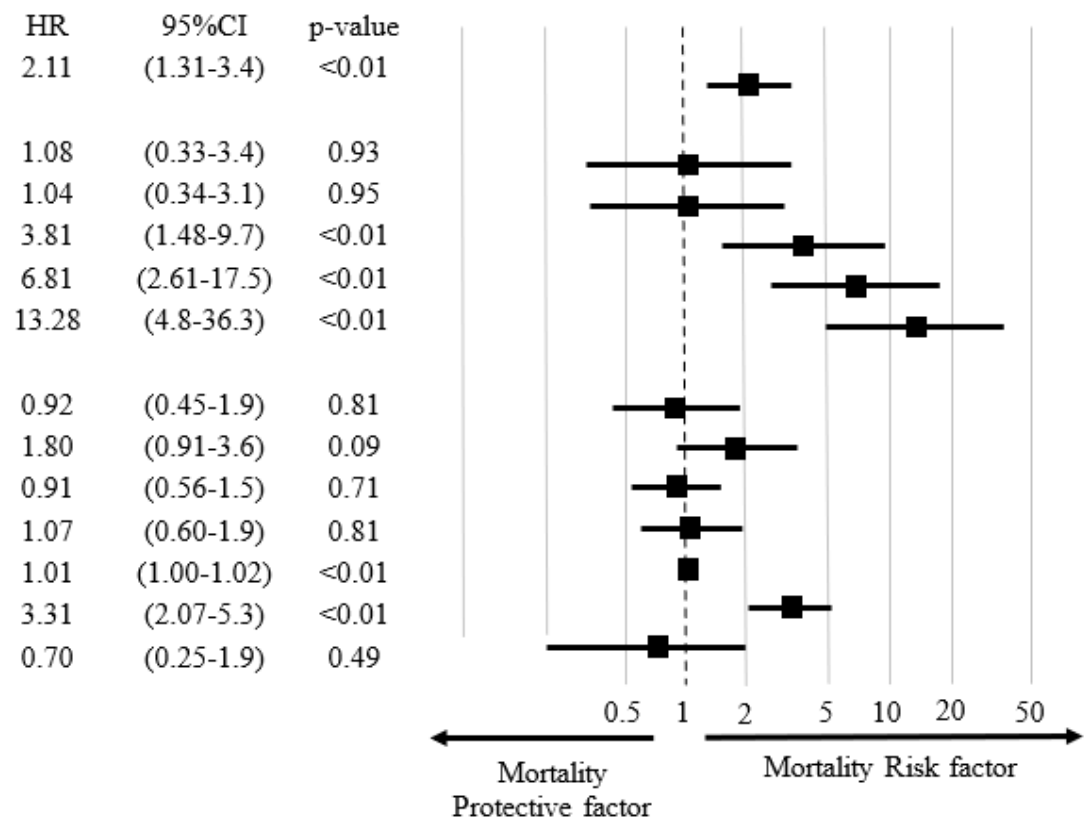

A

$\begin{array}{ccc}\text { HR } & \begin{array}{c}95 \% \mathrm{CI} \\ 2.02\end{array} & \begin{array}{c}\text { p-value } \\ (1.25-3.3)\end{array} \\ & & \\ & & \\ 1.35 & (0.43-4.2) & 0.93 \\ 1.07 & (0.35-3.2) & 0.95 \\ 3.98 & (1.5-10.6) & <0.01 \\ 6.74 & (2.6-17.3) & <0.01 \\ 14.3 & (5.2-39.7) & <0.01 \\ & & \\ 0.93 & (0.45-1.9) & 0.81 \\ 2.62 & (1.3-5.24) & 0.09 \\ 0.89 & (0.55-1.5) & 0.65 \\ 1.04 & (0.60-1.9) & 0.89 \\ 1.01 & (1.00-1.02) & <0.01 \\ 3.71 & (2.32-5.9) & <0.01 \\ 0.91 & (0.33-2.55) & 0.85\end{array}$

Protective factor

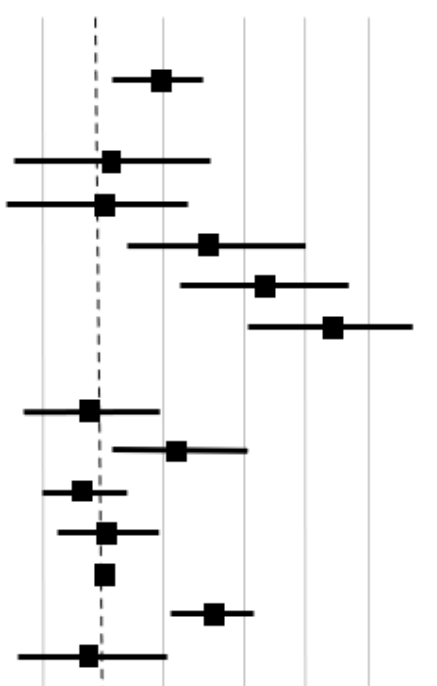

$\begin{array}{lllllll}0.5 & 1 & 2 & 5 & 10 & 20 & 50 \\ \end{array}$ Mortality Risk factor

\section{Figure 3}

a. Forest plot of cox proportional hazard model for in-hospital mortality. $\mathrm{HR}=\mathrm{Hazard}$ Ratio; $\mathrm{Cl}=\mathrm{Confident}$ Interval; DOACs=Direct Oral Anticoagulants, VKA=Vitamin K antagonist, $\mathrm{SD}=$ Standard Deviation, $\mathrm{CT}=$ Computerized Tomography. *Venous thrombosis event included deep vein thrombosis and Pulmonary Embolism. b. Forest plot of cox proportional hazard model for in-hospital mortality in the population without censored outcome $(n=886)$. HR= Hazard Ratio; $\mathrm{Cl=Confident} \mathrm{Interval;} \mathrm{DOACs=Direct} \mathrm{Oral}$ 
Anticoagulants, VKA=Vitamin $\mathrm{K}$ antagonist, $\mathrm{SD}=\mathrm{Standard}$ Deviation, $\mathrm{CT}=$ Computerized Tomography. *Venous thrombosis event included deep vein thrombosis and Pulmonary Embolism. 